\title{
HOM COUPLERS DESIGN FOR THE SUPER-3HC CAVITY
}

\author{
P. Craievich, Sincrotrone Trieste, Italy \\ P. Bosland, S. Chel, M. Juillard, M. Luong, M. Maurier, D. Roudier, CEA Saclay, France
}

\begin{abstract}
We report on the design of high order mode (HOM) couplers developed for superconducting (SC) $3^{\text {rd }}$ harmonic cavities carried out in the framework of the SUPER 3HC project. The high damping requirements for both longitudinal and transverse modes can be achieved with the appropriate HOM dampers. The results of measurements on a copper model as well as simulations are presented.
\end{abstract}

\section{INTRODUCTION}

In an electron storage ring, the enhancement of bunch charge density leads to a beam lifetime reduction [1]. This phenomenon represents an important limitation for today's "Third Generation" high brightness synchrotron radiation sources. A promising way to cure the instabilities and recover a longer lifetime consists in the use of passive SC harmonic cavities [2,3]. There are many possible designs for a HOM-free normal conducting cavity [4,5]. Most of them attempt to couple the HOM power out of the cavity through small slots or antennae implemented at the equator. However, these solutions affect the very sensitive area of the equator and appear inappropriate for a SC cavity. For the latter case, the new scheme initially proposed for the SOLEIL project is more attractive [6]. It combines 2 cells connected with an inner pipe on which monopole and dipole HOM loop-like couplers are mounted. A collaboration, known as the SUPER-3HC project, between SINCROTRONE TRIESTE (Italy), PSI (Switzerland) and CEA-DAPNIA (France) has been approved with the objective of building 2 sets of SC passive harmonic cavities and cryomodules, one for each of the 2 first partners. Detailed information about the collaboration is found in [7]

\section{DESIGN OVERVIEW}

The design of the cavity and its HOM couplers is mainly based on a scaling of the SOLEIL cavity-from $350 \mathrm{MHz}$ to $1500 \mathrm{MHz}$. Fig. 1 shows the arrangement of the HOM couplers on the cavity whose 2 cells are housed inside the helium vessels. The upward "V" is composed of $2 \mathrm{HOM}$ couplers for monopole modes, while the dipole mode couplers form the 2 downward "V". The $115^{\circ}$ angle of the "V" allows an extra coupling of the quadrupole modes. The "classical" configuration of a coaxial line $\left(\phi_{\text {in }}=8 \mathrm{~mm}, \phi_{\text {out }}=40 \mathrm{~mm}\right)$ ending in a loop at the inner pipe is used for the HOM couplers (see Fig. 2). The plane defined by the loop is respectively parallel and orthogonal to the beam axis for the monopole and dipole couplers.

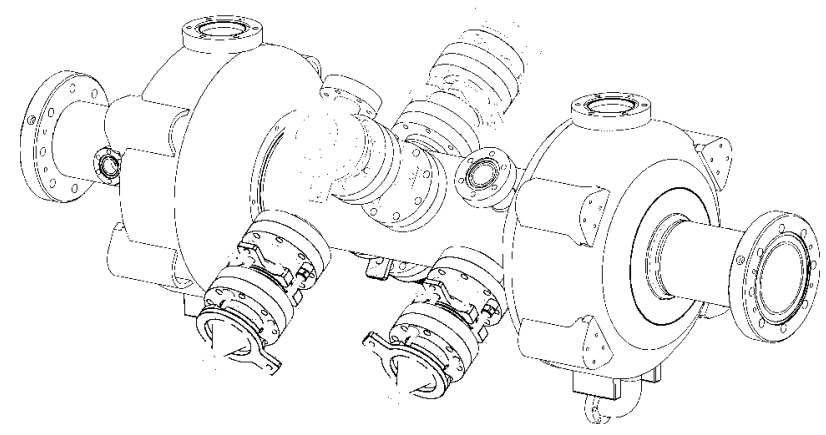

Figure 1: 2 cells Super-3HC cavity with HOM couplers.

Two special thin wall coaxial lines-ensuring low thermal conduction and high power handling - extend the monopole couplers in order to extract out of the cryomodule, a total amount of power estimated at about $1 \mathrm{~kW}$. On dipole couplers, the transported power of about tens watts allows the use of standard semi-rigid cable (Kaman $\phi 0.2$ ") with the adjunction of a conical adapter as shown on Fig. 2.

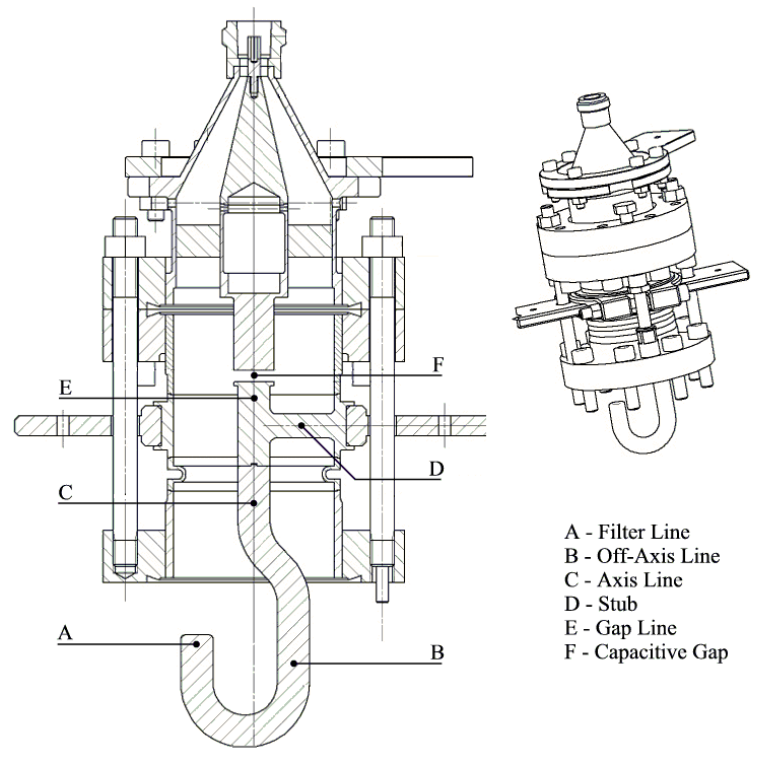

Figure $2:$ HOM coupler (here for the dipole modes).

\section{DAMPING REQUIREMENTS}

The requirements differ from ELETTRA (Sincrotrone Trieste) to SLS (PSI) since the machine parameters are different. Nevertheless, a common agreement has been achieved to specify a maximum longitudinal impedancemode frequency product of $7 \mathrm{k} \Omega . \mathrm{GHz}$ and a transverse impedance of $130 \mathrm{k} \Omega \cdot \mathrm{m}^{-1}$. The characteristics of high 
order modes under the cutoff frequency of the beam pipe $(\phi \quad 61 \mathrm{~mm})$ are calculated with URMEL and the requirements translated in term of maximum external $\mathrm{Q}$ are reported in Table 1.

Table 1 : Damping Requirements

\begin{tabular}{|c|c|r|c|c|r|}
\hline $\mathrm{M}$ & $\mathrm{R}_{/ /} / \mathrm{Q}$ & $\mathrm{Q}_{\max .}$ & $\mathrm{D}$ & $\mathrm{R}_{\perp} / \mathrm{Q}$ & $\mathrm{Q}_{\max .}$ \\
\hline 2466 & 0.17 & 16000 & 1721 & 20 & 6500 \\
\hline 2532 & 2.60 & 1100 & 1723 & 218 & 600 \\
\hline 2067 & 11.0 & 240 & 1935 & 0.01 & $110^{7}$ \\
\hline 2695 & 0.12 & 22000 & 2056 & 255 & 510 \\
\hline 2826 & 6.57 & 380 & 2103 & 27.6 & 4710 \\
\hline 2979 & 8.61 & 270 & 2148 & 437 & 300 \\
\hline 3084 & 1.93 & 1200 & 2303 & 10.1 & 12900 \\
\hline 3180 & 0.30 & 7500 & 2503 & 11.1 & 11700 \\
\hline 3358 & 0.86 & 2400 & 2712 & 63.8 & 2040 \\
\hline 3594 & 0.43 & 4500 & 2865 & 10.3 & 12600 \\
\hline
\end{tabular}

$\mathrm{M}$ for Monopole modes and D for Dipole modes (frequency in $\mathrm{MHz}$ ), $\mathrm{R}_{/ /} / \mathrm{Q}$ and $\mathrm{R}_{\perp} / \mathrm{Q}$ unit is respectively $\Omega$ and $\Omega . \mathrm{m}^{-1}$.

\section{COUPLERS OPTIMIZATION}

The optimization procedure is split into 2 steps. First, the "best" position of the HOM coupler along the inner pipe is calculated with the assumption that the loopshaped coupler has a flat frequency transmittance. Second, the different parts of the coupler-A, B, C, D, E, $\mathrm{F}$ in Fig. 2, as well as the distance of the loop from the beam axis-are optimized by a direct measurements of $\mathrm{Q}$ on a copper model.

\subsection{Position of HOM Coupler on the Inner Pipe}

The optimal position of the coupler corresponds to minimal impedance of all critical modes on a plot where the position of an idealized coupler is varied as a parameter (see Fig. 3). The calculation of the impedance uses an external $\mathrm{Q}$ approximated by $\left(2 \mathrm{R}_{\mathrm{L}} \mathrm{W}\right) /\left(\omega \mu_{0}^{2} \mathrm{~S}^{2}\right.$ $\mathrm{H}_{\mathrm{i}}^{2}$ ), where $\mathrm{i}=\mathrm{z}, \phi$ respectively for dipole and monopole coupler, $R_{L}=50 \Omega$, W the stored energy and $H_{i}$ are given by URMEL for each mode.

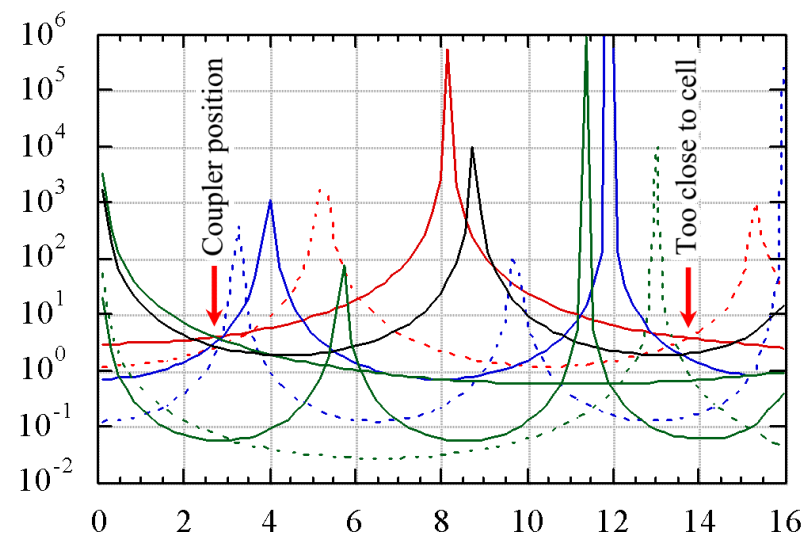

Figure 3 : Longitudinal impedance $(\mathrm{k} \Omega)$ as function of coupler position $(\mathrm{cm})$ from the center of the inner pipe.
After Fig. 3, the monopole couplers are located optimally at $2.7 \mathrm{~cm}$ from the center of the inner pipe. The same calculation is carried out for the dipole couplers giving an optimal position at $10 \mathrm{~cm}$.

\subsection{Coupler Parts Optimization}

This optimization is based on a copper model. The different parts of the coupler are available in various dimensions. Cavity and couplers form a system similar to a construction set. Two small loop antennae placed at the equator of each cell perform a direct measurement (3dB) of the loaded $\mathrm{Q}$, which is a very good approximation of external Q since the unloaded Q is about tens thousands for the copper cavity. The azimuthal position of the antennae can be varied in order to excite both polarizations of the dipole modes. Since the loop-like coupler does not have a broadband transmittance, the optimization aims at getting the lowest $\mathrm{Q}$ for a maximum of modes through a cut-and-try process. The 2 monopole couplers have a different geometry in order to achieve the best damping for all modes. Fig. 4 reports their performances compared to the requirements.

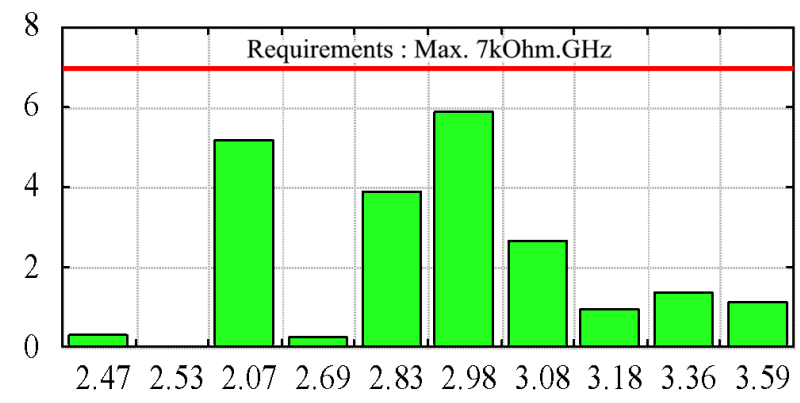

Figure 4 : Longitudinal impedances $(\mathrm{k} \Omega . \mathrm{GHz})$ versus monopole modes (frequency in $\mathrm{GHz}$ )

The 4 dipole mode couplers must be identical because the 2 first dipole modes are under the cutoff frequency $(1869 \mathrm{MHz})$ of the inner pipe and both polarizations have to be damped. For cost and time saving, only 2 identical couplers are used on the copper model in the optimization process. The total external $\mathrm{Q}$ with 4 couplers is assumed to be half of that measured with 2 couplers. For the 2 first dipole modes, measurements are perturbed by the undamped polarization. Their external Q is calculated with HFSS [8]. Final results are shown in Fig. 5.

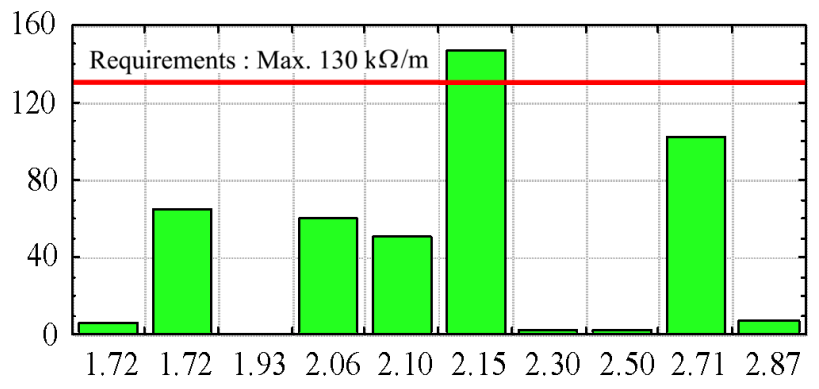

Figure 5 : Transverse impedance $(\mathrm{k} \Omega / \mathrm{m})$ versus dipole modes (frequency in $\mathrm{GHz}$ ) 


\subsection{Rejection of the Fundamental Mode}

The rejection of the fundamental mode $(1500 \mathrm{MHz})$ on the HOM coupler, characterized by the associated external Q, takes advantage of the azimuthal symmetry of the mode. As the coupling loop is not symmetrical, the capacitive gap between the loop end and the external conductor, so called filter-gap, should be adjusted to compensate for this asymmetry. This compensation is more critical for the dipole coupler than for the monopole one, since its loop is orthogonal to the beam axis and closer to the cell. On dipole coupler, a fine tune of the filter-gap capacitance is obtained with a very small tilt of the loop in its plane. The external Q is measured on the copper model by :

$$
\mathrm{Q}_{\mathrm{ext}} \approx \frac{\mathrm{Q}_{0}\left(1-\left.\mathrm{S}_{11}\right|^{2}\right)}{\left|\mathrm{S}_{21}\right|^{2}},
$$

where $\mathrm{Q}_{0}$ is the unloaded $\mathrm{Q}$ of the copper cavity, port 1 the excitation loop and port 2 the HOM coupler. The worst rejection corresponds to $\mathrm{Q}_{\mathrm{ext}}$ of $1.810^{9}$ (result of a compromise with the HOM damping) and $2.310^{10}$ respectively for each monopole and dipole coupler, giving a total external $\mathrm{Q}$ of $7.810^{8}$. Assuming an unloaded $\mathrm{Q}$ of $210^{8}$ for the niobium sputtered cavity, the total Q of the cavity with HOM couplers would be $1.610^{8}$, still higher than the specified lowest limit of $10^{8}$.

\subsection{Sensitivity of the Rejection}

The use of standard coaxial cables to connect the dipole coupler to the outside of the cryomodule makes the tuning of the fundamental mode rejection more critical because of RF dissipation. The sensitivity of this tuning (with regard to the loop angle, the filter-gap, and the filter-line) has to be analyzed. All the investigations could not be carried out easily on the copper model. Therefore, an evaluation with HFSS is produced. Fig. 6 presents the model used in the simulations.



Figure 6 : HFSS model for the sensitivity analysis

The results for the sensitivity to the filter-line length (see Fig. 2), the filter-gap and the loop angle are given respectively in Table 2, 3 and 4 . The lower the value of $\left|\mathrm{S}_{21}\right|$, the better the rejection.
Table 2 : Rejection versus filter line length.

\begin{tabular}{|l|c|c|c|c|c|c|}
\hline $\mathrm{L}(\mathrm{mm})$ & 12 & 11.5 & 11.3 & 11.1 & 11 & 10 \\
\hline$\left|\mathrm{S}_{21}\right| \mathrm{dB}$ & -46 & -51 & -53 & -60 & -61 & -46 \\
\hline
\end{tabular}

Table 3 : Rejection versus filter gap.

\begin{tabular}{|l|c|c|c|c|c|c|}
\hline$\Delta(\mathrm{mm})$ & -0.6 & -0.4 & -0.2 & 0.0 & 0.2 & 0.4 \\
\hline$\left|\mathrm{S}_{21}\right| \mathrm{dB}$ & -33 & -37 & -43 & -63 & -48 & -42 \\
\hline
\end{tabular}

$\Delta=0$ corresponds to the nominal position, gap $=1.8 \mathrm{~mm}$.

Table 4 : Rejection versus loop angle

\begin{tabular}{|c|c|c|c|c|c|c|}
\hline$\alpha\left(^{\circ}\right)$ & +10 & +5 & +2 & 0 & -2 & -5 \\
\hline$\left|S_{21}\right| \mathrm{dB}$ & -32 & -38 & -49 & -63 & -44 & -36 \\
$\alpha=0^{\circ}$ corresponds to theminal position ; loop plane $\perp$ to cavity axis.
\end{tabular}

The data in table 2 could be obtained with measurement on the model. The crosscheck gave good agreement and confidence to the simulation results. Provided a careful manufacturing and mounting of the dipole coupler, a good rejection could be achieved. The high sensitivity to the gap is used as the tuning parameter.

\section{CONCLUSION}

The design of the HOM couplers for the SUPER-3HC cavity is completed. The damping of HOM impedances is fulfilled for all the modes except one, which is only about $15 \%$ above the limit. HFSS turned out to be very accurate for some specific analysis even though all the optimization process could not be based on it because of the computer resources and speed limitation at the time being.

\section{REFERENCES}

[1] B. Touschek et al., "Lifetime and Beam size in a Storage Ring", Phys. Rev. Letters, 1963

[2] M. Svandrlik et al., "Design of a $3^{\text {rd }}$ Harmonic SC Cavity for Bunch Lengthening in ELETTRA", EPAC'98, Stockholm, June 1998

[3] P. Marchand, "Possible Upgrading of the SLS RF System for Improving the Beam Lifetime", PAC'99, New York, March 1999

[4] T. Takahashi et al., "Design of a HOM Coupler for a Damped Cavity at the Photon Factory Storage Ring", PAC'99, New York, March 1999

[5] C. Yinghua et al., "The Slotted Cavity-a Method of Broadband Damping", Proceedings of the XIV International Conference on High Energy Accelerators, Tsukuba, 1989

[6] A. Mosnier et al., "Design of a Heavily Damped Superconducting Cavity for SOLEIL", PAC'97, Vancouver, 1997

[7] M. Svandrlik et al., "The Super-3HC Project : an Idle Superconducting Harmonic Cavity for Bunch Length Manipulation", EPAC'2000, Vienna, 2000

[8] HFSS - High Frequency Structure Simulation code, from Agilent Technologies. 